\title{
Second-Order Differential Operators with Non-Local Ventcel's Boundary Conditions
}

\author{
MICHELE CAMPITI*
}

\begin{abstract}
Different boundary conditions have been introduced for second-order differential operators and the properties of the operators on the corresponding domains have been deeply investigated since the work of Feller. The aim of this paper is to study second-order differential operators satisfying a Ventcel's type boundary condition which involves simultaneously both the endpoints of a real interval. We study different general properties and a resolvent estimate for this kind of operators.
\end{abstract}

Keywords: Ventcel's Type Boundary Conditions, Second-Order Differential Operators, Boundary Conditions 2010 Mathematics Subject Classification: 47D06, 34B05

\section{INTRODUCTION AND NOTATION}

Let $-\infty \leq r_{1}<r_{2} \leq+\infty$, set $\left.I:=\right] r_{1}, r_{2}[$ and consider the second-order differential operator

$$
A u(x)=\alpha(x) u^{\prime \prime}(x)+\beta(x) u^{\prime}(x), \quad x \in I,
$$

where $\alpha, \beta \in C(\bar{I})$ and $\alpha(x)>0$ for every $x \in I$.

Using the work of Feller [7], several authors have characterized the generation of a $C_{0}$-semigroup studying particular boundary conditions on the operator $A$, such as Ventcel's boundary conditions [5] on the corresponding domain

$$
D_{V}(A)=\left\{u \in C(\bar{I}) \cap C^{2}(I) \mid \lim _{x \rightarrow r_{1}} A u(x)=0 \text { and } \lim _{x \rightarrow r_{2}} A u(x)=0\right\},
$$

or the maximal domain [9]

$$
D_{M}(A)=\left\{u \in C(\bar{I}) \cap C^{2}(I) \mid A u \in C(\bar{I})\right\} .
$$

We also point out that further characterizations have been obtained in $L^{1}(I)$ for different domains [3] and even for periodic functions [4].

In this paper, we consider a different boundary condition which involves the two endpoints simultaneously. Namely, we fix a real parameter $\rho \neq 0$ and define

$$
D_{\rho}(A):=\left\{u \in D_{M}(A) \mid A u\left(r_{1}\right)+\rho A u\left(r_{2}\right)=0\right\} .
$$

Obviously

$$
D_{V}(A) \subset D_{\rho}(A) \subset D_{M}(A),
$$

and this implies that $\left(A, D_{\rho}(A)\right)$ is densely defined in $C(\bar{I})$.

Received: 9 June 2019; Accepted: 26 July 2019; Published Online: 31 July 2019

*Corresponding author: M. Campiti; michele.campiti@unisalento.it

DOI: $10.33205 / \mathrm{cma} .574194$ 
Our aim is to study some properties of the operator $\left(A, D_{\rho}(A)\right)$ in $C(\bar{I})$. These conditions are expressed in terms of Feller's classification of the boundary points and therefore we make the following recalls. Fix $x_{0} \in I$ and define, for every $x \in I$,

$$
W(x):=\exp \left(-\int_{x_{0}}^{x} \frac{\beta}{\alpha}\right), Q(x):=\frac{1}{\alpha(x) W(x)} \int_{x_{0}}^{x} W, R(x):=W(x) \int_{x_{0}}^{x} \frac{1}{\alpha W} .
$$

According to the classification introduced by Feller [7] (see also [6, p. 396]), the endpoint $r_{2}$ is

$$
\begin{array}{lll}
\text { a regular boundary } & \text { if } & Q \in L^{1}(] x_{0}, r_{2}[), R \in L^{1}(] x_{0}, r_{2}[) ; \\
\text { an exit boundary } & \text { if } & Q \notin L^{1}(] x_{0}, r_{2}[), R \in L^{1}(] x_{0}, r_{2}[) ; \\
\text { an entrance boundary } & \text { if } & Q \in L^{1}(] x_{0}, r_{2}[), R \notin L^{1}(] x_{0}, r_{2}[) ; \\
\text { a natural boundary } & \text { if } & Q \notin L^{1}(] x_{0}, r_{2}[), R \notin L^{1}(] x_{0}, r_{2}[)
\end{array}
$$

The classification of $r_{1}$ is similar with $] r_{1}, x_{0}[$ in place of $] x_{0}, r_{2}[$.

Boundary conditions involving simultaneously the two endpoints arise in many differential problems, such as oscillations under external and resistant forces or the working of kidneys in biology (see also [8, Sections IV.3 and V.2] and [7, Section 23] for some related discussions).

\section{GENERAL PROPERTIES OF NON-LOCAL BOUNDARY CONDITIONS.}

We begin with some preliminary properties of the operator $\left(A, D_{\rho}(A)\right)$. Some straightforward details given in [7] and also in [5,9] will be briefly outlined.

Lemma 2.1. The operator $\left(A, D_{\rho}(A)\right)$ is closed.

Proof. Let $u \in D_{\rho}(A)$ and $\left.[a, b] \subset\right] r_{1}, r_{2}\left[\right.$. If $\varepsilon>0$ there exists $K_{\varepsilon}>0$ such that $\left|u^{\prime}(x)\right| \leq$ $\varepsilon\left\|u^{\prime \prime}\right\|_{[a, b]}+K_{\varepsilon}\|u\|_{[a, b]}$ for every $x \in[a, b]$. Since $u \in C^{2}([a, b])$ and $\beta$ is continuous, we can find $C_{\varepsilon}>0$ such that $\left|\beta(x) u^{\prime}(x)\right| \leq \varepsilon\left\|u^{\prime \prime}\right\|_{[a, b]}+C_{\varepsilon}\|u\|_{[a, b]}$.

Since $\alpha>0$ on $[a, b]$ there exists $C>0$ such that $\left\|u^{\prime \prime}\right\|_{[a, b]} \leq C\left(\|A u\|_{[a, b]}+\|u\|_{[a, b]}\right)$. Now, consider a sequence $\left(u_{n}\right)$ in $D_{\rho}(A)$ which converges uniformly to $u \in C\left(\left[r_{1}, r_{2}\right]\right)$ and such that $\left(A u_{n}\right)$ converges uniformly to $v \in C\left(\left[r_{1}, r_{2}\right]\right)$. We have only to show that $u \in D_{\rho}(A)$ and $A u=v$.

If $[a, b] \subset] r_{1}, r_{2}\left[\right.$, from the preceding estimates we have that $\left(u_{n}^{\prime \prime}\right)$ is a Cauchy sequence in $C([a, b])$ and therefore it is uniformly convergent in $C([a, b])$ and analogously, we have also the uniform convergence in $C([a, b])$ of the sequence $\left(u_{n}^{\prime}\right)$. We conclude that $u \in C^{2}([a, b])$ and $A u=v$ on $[a, b]$. Since $[a, b]$ is arbitrarily chosen, we get $u \in C\left(\left[r_{1}, r_{2}\right]\right) \cap C^{2}(] r_{1}, r_{2}[)$ and $A u=v$. Finally, the boundary condition $\lim _{x \rightarrow r_{1}} A u(x)+\rho \lim _{x \rightarrow r_{2}} A u(x)=0$ is a consequence of the uniform convergence of $\left(A u_{n}\right)$ to $A u$ and $u_{n} \in D_{\rho}(A)$.

Let $\lambda>0, x_{0} \in I$ and denote by $\Sigma_{\lambda}\left(x_{0}\right)$ the set of all solutions $u \in C(\bar{I}) \cap C^{2}(I)$ of $\lambda u-A u=0$ satisfying $u\left(x_{0}\right)=1$. Moreover, let

$$
\begin{aligned}
& A_{1, \lambda}\left(x_{0}\right)=\left\{u \in \Sigma_{\lambda}\left(x_{0}\right) \mid u \text { is positive and increasing }\right\}, \\
& A_{2, \lambda}\left(x_{0}\right)=\left\{u \in \Sigma_{\lambda}\left(x_{0}\right) \mid u \text { is positive and decreasing }\right\} .
\end{aligned}
$$

From [7, Sections 8 and 9] and [5] there exist $\chi_{1, \lambda} \in A_{1, \lambda}\left(x_{0}\right)$ and $\chi_{2, \lambda} \in A_{2, \lambda}\left(x_{0}\right)$ such that

(1) If $u \in A_{1, \lambda}\left(x_{0}\right)$ we have $u \geq \chi_{1, \lambda}$ on $\left[r_{1}, x_{0}\right]$ and $u \leq \chi_{1, \lambda}$ on $\left[x_{0}, r_{2}\right]$.

(2) If $u \in A_{2, \lambda}\left(x_{0}\right)$ we have $u \leq \chi_{2, \lambda}$ on $\left[r_{1}, x_{0}\right]$ and $u \geq \chi_{2, \lambda}$ on $\left[x_{0}, r_{2}\right]$.

Moreover, if we put

$$
\gamma_{i, \lambda}:=\lim _{x \rightarrow r_{i}} \chi_{i, \lambda}(x)\left(=\inf \chi_{i, \lambda}\right), \quad M_{i, \lambda}:=\lim _{x \rightarrow r_{3-i}} \chi_{i, \lambda}(x)\left(=\sup \chi_{i, \lambda}\right), \quad i=1,2,
$$


we have ([7, Lemma 9.3] and [5, Lemma 5 and Lemma 7])

$$
\begin{aligned}
\gamma_{1, \lambda}>0 & \Leftrightarrow W \notin L^{1}\left(r_{1}, x_{0}\right) \text { and } \int_{r_{1}}^{x_{0}} W(x) \int_{r_{1}}^{x} \frac{1}{\alpha(t) W(t)} d t d x<+\infty, \\
\gamma_{2, \lambda}>0 & \Leftrightarrow W \notin L^{1}\left(x_{0}, r_{2}\right) \text { and } \int_{x_{0}}^{r_{2}} W(x) \int_{x}^{r_{2}} \frac{1}{\alpha(t) W(t)} d t d x<+\infty,
\end{aligned}
$$

that is, $r_{1}$ (respectively $r_{2}$ ) is not an entrance boundary.

Finally, in [9, Lemma 6] it has been shown that

$$
M_{1, \lambda}<+\infty \Leftrightarrow \int_{x_{0}}^{r_{2}} R(x) d x<+\infty, \quad M_{2, \lambda}<+\infty \Leftrightarrow \int_{r_{1}}^{x_{0}} R(x) d x<+\infty,
$$

which means that $r_{1}$ (respectively $r_{2}$ ) is not entrance nor natural.

If $u_{i} \in A_{i, \lambda}\left(x_{0}\right), i=1,2$, we can define the Green's function

$$
G_{u_{1}, u_{2}}(x, s):= \begin{cases}\frac{u_{1}(x) u_{2}(s)}{\alpha(s) v(s)}, & r_{1}<x \leq s<r_{2}, \\ \frac{u_{1}(x) u_{2}(s)}{\alpha(s) v(s)}, & r_{1}<s<x<r_{2},\end{cases}
$$

where $v(s):=u_{1}^{\prime}(s) u_{2}(s)-u_{2}^{\prime}(s) u_{1}(s)$. Then for every $f \in C(\bar{I})$, the function

$$
\left.u_{f}(x):=\int_{r_{1}}^{r_{2}} G_{u_{1}, u_{2}}(x, s) f(s) d s, \quad x \in\right] r_{1}, r_{2}[
$$

is a solution of $\lambda u_{f}-A u_{f}=f$ satisfying $\lambda\left\|u_{f}\right\| \leq\|f\|$ and is positive whenever $f$ is positive (see [7, Theorem 13.1]); if the endpoint $r_{i}, i=1,2$, is a regular or an exit point, we also have $\lim _{x \rightarrow r_{i}} u_{f}(x)=0$ (see [8, \#8, p. 32]).

Consequently the general solution of $\lambda u-A u=f$ (on $I$ ) is given by

$$
u_{f}\left(x ; \lambda, c_{1}, c_{2}\right)=u_{f}(x)+c_{1} \chi_{1, \lambda}(x)+c_{2} \chi_{2, \lambda}(x), \quad x \in I .
$$

If one of the endpoints is natural, then $D_{\rho}(A)=D_{V}(A)$. Indeed, from [7, Corollary to Theorem 13.1], the Ventcel's boundary is always satisfied at a natural endpoint and consequently the non local boundary condition (1.2) implies the Ventcel's boundary condition at the other endpoint too. In this case $\left(A, D_{\rho}(A)\right)$ generates a $C_{0}$-semigroup if and only if the other endpoint is not an entrance boundary [5, Theorem 2].

In the case of two entrance boundary points, we cannot prescribe the values of $A u$ at any endpoint and therefore we have the generation of a $C_{0}$-semigroup only on the maximal domain. To avoid these well-known cases, in the sequel we shall require

$$
D_{V}(A) \underset{f}{\subset} D_{\rho}(A) \underset{\neq}{\subset} D_{M}(A),
$$

which implies that each endpoint is not a natural boundary and that the two endpoints are not both entrance boundaries.

We shall find the most interesting properties in the case where one of the endpoints is an entrance boundary and the other a regular or exit boundary.

We shall require that the integral of $R$ will be finite only on just one of the intervals $\left[r_{1}, x_{0}\right]$ and $\left[x_{0}, r_{2}\right]$, that is

$$
\int_{x_{0}}^{r_{1}} R(x) d x<+\infty, \quad \int_{x_{0}}^{r_{2}} R(x) d x=+\infty
$$

or

$$
\int_{x_{0}}^{r_{1}} R(x) d x=+\infty, \quad \int_{x_{0}}^{r_{2}} R(x) d x<+\infty
$$


Remark 2.1. Assume that (2.7) is satisfied. Then, conditions (2.8)-(2.9) hold if and only if one boundary point is entrance and the other is regular or exit.

Indeed, changing the order of integration, we can write

$$
\int_{x_{0}}^{r_{1}} R(x) d x=\int_{r_{1}}^{x_{0}} \frac{1}{\alpha(x) W(x)} \int_{r_{1}}^{x} W(t) d t d x
$$

and

$$
\int_{x_{0}}^{r_{2}} R(x) d x=\int_{x_{0}}^{r_{2}} \frac{1}{\alpha(x) W(x)} \int_{x}^{r_{2}} W(t) d t d x .
$$

If $\int_{x_{0}}^{r_{1}} R(x) d x<+\infty$, we must have $W \in L\left(r_{1}, x_{0}\right)$; therefore, according to [7, Section 23], if $\frac{1}{\alpha W} \in L\left(r_{1}, x_{0}\right)$ then $r_{1}$ is a regular boundary point while if $\frac{1}{\alpha W} \notin L\left(r_{1}, x_{0}\right)$ then $r_{1}$ is an exit boundary point.

Conversely, it is clear that if $r_{1}$ is a regular or exit boundary point, then necessarily $\int_{x_{0}}^{r_{1}} R(x) d x<$ $+\infty$ and thus condition $\int_{x_{0}}^{r_{1}} R(x) d x=+\infty$ implies that $r_{1}$ is an entrance or natural boundary point. An analogous reasoning holds at the point $r_{2}$.

If (2.8) holds, we must have

$$
\gamma_{1, \lambda}=0, \quad M_{1, \lambda}=+\infty, \quad \gamma_{2, \lambda}>0, \quad M_{2, \lambda}<+\infty
$$

and similarly, if (2.9) holds,

$$
\gamma_{1, \lambda}>0, \quad M_{1, \lambda}<+\infty, \quad \gamma_{2, \lambda}=0, \quad M_{2, \lambda}=+\infty ;
$$

indeed, condition (2.8) is equivalent to $M_{1, \lambda}=+\infty$ and $M_{2, \lambda}<+\infty$ and (2.9) to $M_{1, \lambda}<+\infty$ and $M_{2, \lambda}=+\infty$.

Moreover, if (2.8) holds, again by (2.10) it follows that there exists no decreasing positive solution of $\lambda u-A u=0$ which is independent of $\chi_{2, \lambda}$. Hence, as in [8, pp. 25-37], we can obtain a different expression of $\chi_{2, \lambda}$; namely, for $n \in \mathbf{N}$ and $x \in I$ we define recursively

$$
u_{0}(x)=1, \quad u_{n+1}(x)=\int_{x_{0}}^{x} W(t) \int_{x_{0}}^{t} \frac{u_{n}(s)}{\alpha(s) W(s)} d s d t
$$

and we put

$$
u(x, \lambda):=\sum_{n=0}^{+\infty} \lambda^{n} u_{n}(x) .
$$

A decreasing positive solution of $\lambda u-A u=0$ is [8, pp. 26-27]

$$
u_{+}(x, \lambda):=u(x, \lambda) \int_{x}^{r_{2}} \frac{W(t)}{u(t, \lambda)^{2}} d t
$$

and normalizing both solutions $\chi_{2, \lambda}$ and $u_{+}(\cdot, \lambda)$, we obtain

$$
\chi_{2, \lambda}(x)=M_{2, \lambda} \frac{u_{+}(x, \lambda)}{u_{+}\left(r_{1}, \lambda\right)}, \quad x \in I .
$$

Similarly, as a consequence of (2.9), it is possible to show that

$$
\left.\chi_{1, \lambda}(x)=M_{1, \lambda} \frac{u_{-}(x, \lambda)}{u_{-}\left(r_{2}, \lambda\right)}, \quad x \in\right] r_{1}, r_{2}[,
$$

where

$$
u_{-}(x, \lambda):=u(x, \lambda) \int_{r_{1}}^{x} \frac{W(t)}{u(t, \lambda)^{2}} d t
$$


Taking into account the above discussion, we can state the following result.

Proposition 2.1. Assume that condition (2.8) holds. Then, the map $\lambda \mapsto \gamma_{2, \lambda} / M_{2, \lambda}$ is continuous and strictly decreasing and further

$$
\lim _{\lambda \rightarrow 0^{+}} \frac{\gamma_{2, \lambda}}{M_{2, \lambda}}=1, \quad \lim _{\lambda \rightarrow+\infty} \frac{\gamma_{2, \lambda}}{M_{2, \lambda}}=0 .
$$

Analogously, if (2.9) holds, then the map $\lambda \mapsto \gamma_{1, \lambda} / M_{1, \lambda}$ is continuous and strictly decreasing and

$$
\lim _{\lambda \rightarrow 0^{+}} \frac{\gamma_{1, \lambda}}{M_{1, \lambda}}=1, \quad \lim _{\lambda \rightarrow+\infty} \frac{\gamma_{1, \lambda}}{M_{1, \lambda}}=0 .
$$

Proof. We prove only the first part under the assumption that (2.8) holds. First, observe that the function $\lambda \mapsto \gamma_{2, \lambda} / M_{2, \lambda}$ is well-defined by (2.10) and takes its values in the interval ]0,1[. Its continuity is a direct consequence of (2.12) and (2.13). To show the remaining properties, it is useful to consider the function $u_{2}(x, \lambda)=u_{+}(x, \lambda) / u_{+}\left(r_{1}, \lambda\right)$ and observe that $u_{2}\left(r_{2}, \lambda\right)=$ $\gamma_{2, \lambda} / M_{2, \lambda}$. Since $u_{2}(x, \lambda)$ is a solution of $\lambda u-A u=0$, for every $x \in I$ we have

$$
\lambda=\frac{\alpha(x) u_{2}^{\prime \prime}(x, \lambda)+\beta(x) u_{2}^{\prime}(x, \lambda)}{u_{2}(x, \lambda)}=\frac{u_{2}^{\prime}(x, \lambda)}{u_{2}(x, \lambda)}\left(\alpha(x) \frac{u_{2}^{\prime \prime}(x, \lambda)}{u_{2}^{\prime}(x, \lambda)}+\beta(x)\right) .
$$

If $\lambda \rightarrow 0^{+}$we must have

$$
\frac{u_{2}^{\prime}(x, \lambda)}{u_{2}(x, \lambda)} \rightarrow 0 \quad \text { or } \quad \alpha(x) \frac{u_{2}^{\prime \prime}(x, \lambda)}{u_{2}^{\prime}(x, \lambda)}+\beta(x) \rightarrow 0 .
$$

In the first case, we obtain

$$
\left|u_{2}^{\prime}(x, \lambda)\right| \leq\left|\frac{u_{2}^{\prime}(x, \lambda)}{u_{2}(x, \lambda)}\right| \rightarrow 0
$$

and hence $u_{2}^{\prime}(x, \lambda) \rightarrow 0$ for every $\left.x \in\right] r_{1}, r_{2}\left[\right.$; then, it follows that $\lim _{\lambda \rightarrow 0^{+}} u_{2}(x, \lambda)=1$ and in particular

$$
\lim _{\lambda \rightarrow 0^{+}} \frac{\gamma_{2, \lambda}}{M_{2, \lambda}}=\lim _{\lambda \rightarrow 0^{+}} u_{2}\left(r_{2}, \lambda\right)=1 .
$$

In the second case, we should have that $\lim _{\lambda \rightarrow 0^{+}} u_{2}^{\prime \prime}(x, \lambda) / u_{2}^{\prime}(x, \lambda)=-\beta(x) / \alpha(x)$ from which $\lim _{\lambda \rightarrow 0^{+}} u_{2}^{\prime}(x, \lambda)=c W(x)$ for a suitable constant $c$ and consequently

$$
\lim _{\lambda \rightarrow 0^{+}} u_{2}(x, \lambda)=c_{1}+c_{2} \int_{x_{0}}^{x} W(t) d t .
$$

Since the term $\int_{x_{0}}^{x} W(t) d t$ is unbounded at $r_{2}$, the only possibility is that $c_{2}=0$ and consequently $c_{1}=1$ by evaluation at $r_{1}$; so, we obtain again $\lim _{\lambda \rightarrow 0^{+}} u_{2}(x, \lambda)=1$.

If $\lambda \rightarrow+\infty$, by the equality $\lambda=\alpha(x) u_{2}^{\prime \prime}(x, \lambda) / u_{2}(x, \lambda)+\beta(x) u_{2}^{\prime}(x, \lambda) / u_{2}^{\prime}(x, \lambda)$, we deduce that necessarily $u_{2}(x, \lambda) \rightarrow 0$ at least at one point (and hence at every point by monotonicity). Otherwise, since the functions $\alpha$ and $\beta$ are bounded, at every point we should have that $u_{2}^{\prime}(x, \lambda) \rightarrow$ $-\infty$ or $\left|u_{2}^{\prime \prime}(x, \lambda)\right| \rightarrow+\infty$. It follows that the set $X=\{x \in] r_{1}, r_{2}\left[\mid \lim _{\lambda \rightarrow+\infty} u_{2}^{\prime}(x, \lambda)=-\infty\right\}$ is dense in $] r_{1}, r_{2}\left[\right.$; for, if $[x, y] \cap X=\emptyset$ with $x<y$, then by the equality $u_{2}^{\prime}(y, \lambda)-u_{2}^{\prime}(x, \lambda)=$ $u_{2}^{\prime \prime}\left(t_{\lambda}, \lambda\right)(y-x)$ we get a contradiction by taking a cluster point $t$ for $\left(t_{\lambda}\right)_{\lambda>0}$ where we should have $\lim _{\lambda \rightarrow+\infty}\left|u_{2}^{\prime \prime}(t, \lambda)\right|=+\infty$. At this point, it is clear that $\lim _{\lambda \rightarrow+\infty} u_{2}(x, \lambda)=0$ for every $x \in] r_{1}, r_{2}$ [ and in particular, by the continuity of the map $\lambda \mapsto u_{2}(\cdot, \lambda)$, we conclude that

$$
\lim _{\lambda \rightarrow+\infty} \frac{\gamma_{2, \lambda}}{M_{2, \lambda}}=\lim _{\lambda \rightarrow+\infty} u_{2}\left(r_{2}, \lambda\right)=0
$$

Finally, we have to show that $\left(u_{2}\left(r_{2}, \lambda\right)\right)_{\lambda>0}$ is decreasing. 
Let $0<\lambda<\mu$; then, for every $x \in] r_{1}, r_{2}$ [ we have

$$
\left(\frac{u_{2}^{\prime}(x, \mu)}{W(x)}\right)^{\prime} \frac{1}{u_{2}(x, \mu)}-\left(\frac{u_{2}^{\prime}(x, \lambda)}{W(x)}\right)^{\prime} \frac{1}{u_{2}(x, \lambda)}=\mu-\lambda>0
$$

and consequently we have

$$
\left(\frac{u_{2}^{\prime}(x, \lambda)}{W(x)}\right)^{\prime} \frac{1}{u_{2}(x, \lambda)}<\left(\frac{u_{2}^{\prime}(y, \mu)}{W(y)}\right)^{\prime} \frac{1}{u_{2}(y, \mu)}
$$

for $x, y$ in a suitable interval $] r_{2}-\delta_{1}, r_{2}\left[\right.$. By contradiction, assume that $u_{2}\left(r_{2}, \lambda\right)<u_{2}\left(r_{2}, \mu\right)$; we can choose $\delta_{2}>0$ such that $u_{2}(x, \lambda)<u_{2}(y, \mu)$ for $\left.x, y \in\right] r_{2}-\delta_{2}, r_{2}\left[\right.$. Now, let $\delta=\min \left\{\delta_{1}, \delta_{2}\right\}$; since $u_{2}(\cdot, \lambda) / W$ tends to 0 at $r_{2}$ for every $\lambda>0$ (see, e.g., [7, Section 11, III, p. 488]), for every $x \in] r_{2}-\delta, r_{2}[$ we can write

$$
\frac{u_{2}^{\prime}(x, \lambda)}{W(x)}=\left(\frac{u_{2}^{\prime}(\cdot, \lambda)}{W}\right)^{\prime}(t)\left(x-r_{2}\right), \frac{u_{2}^{\prime}(x, \mu)}{W(x)}=\left(\frac{u_{2}^{\prime}(\cdot, \mu)}{W}\right)^{\prime}(s)\left(x-r_{2}\right)
$$

for suitable $t, s \in] r_{2}-\delta, r_{2}[$ and hence, by the above inequalities, we obtain

$$
u_{2}^{\prime}(x, \lambda) / W(x)>u_{2}^{\prime}(x, \mu) / W(x) \text {, i.e., } u_{2}^{\prime}(x, \lambda)>u_{2}^{\prime}(x, \mu) .
$$

We observe that the inequality $u_{2}^{\prime}(x, \lambda)>u_{2}^{\prime}(x, \mu)$ cannot hold for every $\left.x \in\right] r_{1}, r_{2}[$, since $u_{2}^{\prime}\left(r_{1}, \lambda\right)=u_{2}^{\prime}\left(r_{1}, \mu\right)=1$. Hence we could not have $u_{2}\left(r_{2}, \lambda\right)<u_{2}\left(r_{2}, \mu\right)$. Then, we can find $\left.x_{1} \in\right] r_{1}, r_{2}$ [ for which $u_{2}^{\prime}\left(x_{1}, \lambda\right)=u_{2}^{\prime}\left(x_{1}, \mu\right)$ and $u_{2}^{\prime}(x, \lambda)>u_{2}^{\prime}(x, \mu)$ for every $\left.x \in\right] x_{1}, r_{2}[$. Finally, consider the function $u=u_{2}(\cdot, \mu)-u_{2}(\cdot, \lambda) ; u$ is strictly decreasing and positive on $\left[x_{1}, r_{2}\right]$. Consequently, we have $u^{\prime}\left(x_{1}\right)=0, u^{\prime \prime}\left(x_{1}\right) \leq 0$ and hence $A u\left(x_{1}\right) \leq 0$; on the other hand $A u\left(x_{1}\right)=$ $A u_{2}\left(x_{1}, \mu\right)-A u_{2}\left(x_{1}, \lambda\right)=\mu u_{2}\left(x_{1}, \mu\right)-\lambda u_{2}\left(x_{1}, \lambda\right)>\lambda\left(u_{2}\left(x_{1}, \mu\right)-u_{2}\left(x_{1}, \lambda\right)\right)=\lambda u\left(x_{1}\right)>0$ which is a contradiction. So, the proof is complete.

Theorem 2.1. Assume that $\rho \neq 0$ and that $\left(A, D_{\rho}(A)\right)$ satisfies condition (2.7). Then the following statements are equivalent:

a) There exists $\lambda_{0} \geq 0$ such that $\lambda-A$ is injective for every $\lambda>\lambda_{0}$.

b) $\left(A, D_{\rho}(A)\right)$ satisfies condition (2.8) or (2.9).

Moreover, if condition (2.8) holds, we can take $\lambda_{0}=0$ if and only if $\rho \in[-1,+\infty$ [ while, if condition (2.9) holds, we can take $\lambda_{0}=0$ if and only if $\left.\left.\rho \in\right]-\infty,-1\right] \cup[0,+\infty[$.

Proof. For every $\lambda>0$ the general solution in $I$ of $\lambda u-A u=0$ is given by (see (2.6)) $u_{0}\left(\cdot ; \lambda, c_{1}, c_{2}\right)=$ $c_{1} \chi_{1, \lambda}+c_{2} \chi_{2, \lambda}$, with $c_{1}, c_{2} \in \mathbb{R}$.

We discuss the existence of a unique solution in $D_{\rho}(A)$ on the finiteness of $M_{1, \lambda}$ and $M_{2, \lambda}$. The case $M_{1}=M_{2}=+\infty$ cannot occur otherwise both the endpoints are of entrance or natural type (see [9, Lemma 6]) and this is excluded by condition (2.7).

If condition (2.8) holds, we have $M_{1, \lambda}=+\infty$ and $M_{2, \lambda}<+\infty$ and the general solution in $\bar{I}$ of $\lambda u-A u=0$ is $u_{0}:=c_{2} \chi_{2, \lambda}$ with $c_{2} \in \mathbb{R}$. Moreover $u_{0} \in D_{\rho}(A)$ if and only if $c_{2}\left(M_{2, \lambda}+\rho \gamma_{2, \lambda}\right)=$ 0 . The term $M_{2, \lambda}+\rho \gamma_{2, \lambda}$ may vanish only if $\rho<-1$ and in this case (see Proposition 2.1) there exists $\lambda_{0}>0$ such that $\rho=-M_{2, \lambda_{0}} / \gamma_{2, \lambda_{0}}$. It follows $\gamma_{2, \lambda} / M_{2, \lambda}<\gamma_{2, \lambda_{0}} / M_{2, \lambda_{0}}$ for every $\lambda>\lambda_{0}$ and therefore $M_{2, \lambda}+\rho \gamma_{2, \lambda}>0$. Hence, for every $\lambda>\lambda_{0}$, we must have $c_{2}=0$ and we get that the unique solution of $\lambda u-A u=0$ in $D_{\rho}(A)$ is $u_{0}=0$. If $\rho \geq-1$ we have $M_{2, \lambda}+\rho \gamma_{2, \lambda}>0$ for every $\lambda>0$ and this yields again $c_{2}=0$ and the unique solution $u_{0}=0$ of $\lambda u-A u=0$ in $D_{\rho}(A)$.

If condition (2.9) holds, we have $M_{1, \lambda}<+\infty$ and $M_{2, \lambda}=+\infty$ and the general solution in $\bar{I}$ of $\lambda u-A u=0$ is $u_{0}:=c_{1} \chi_{1, \lambda}$ with $c_{1} \in \mathbb{R}$. Moreover $u_{0} \in D_{\rho}(A)$ if and only if $c_{1}\left(\gamma_{1, \lambda}+\right.$ $\left.\rho M_{1, \lambda}\right)=0$. In this case the term $\gamma_{1, \lambda}+\rho M_{1, \lambda}$ may vanish only if $-1<\rho<0$ and in this case (Proposition 2.1) there exists just one value $\lambda_{0}>0$ such that $\rho=-\gamma_{1, \lambda_{0}} / M_{1, \lambda_{0}}$. It follows 
$\gamma_{1, \lambda} / M_{1, \lambda}<\gamma_{1, \lambda_{0}} / M_{1, \lambda_{0}}$ for every $\lambda>\lambda_{0}$ and consequently $\gamma_{1, \lambda}+\rho M_{1, \lambda}>0$. Thus $c_{1}=0$ and we get the unique solution $u_{0}=0$ of $\lambda u-A u=0$ in $D_{\rho}(A)$. If $\left.\rho \notin\right]-1,0[$ we have $\gamma_{1, \lambda}+\rho M_{1, \lambda}>0$ for every $\lambda>0$ and again $c_{1}=0$ which implies that $u_{0}=0$ is the unique solution of $\lambda u-A u=0$ in $D_{\rho}(A)$.

If $M_{1, \lambda}$ and $M_{2, \lambda}$ are both finite, the general solution in $\bar{I}$ of $\lambda u-A u=0$ is $u_{0}:=c_{1} \chi_{1, \lambda}+c_{2} \chi_{1, \lambda}$ with $c_{1}, c_{2} \in \mathbb{R}$ and we have $u_{0} \in D_{\rho}(A)$ if and only if $c_{1}\left(\gamma_{1, \lambda}+\rho M_{1, \lambda}\right)+c_{2}\left(M_{2, \lambda}+\rho \gamma_{2, \lambda}\right)=0$. In this case we may always obtain infinite solutions in $D_{\rho}(A)$ by taking

$$
c_{1}:=-\frac{M_{2, \lambda}+\rho \gamma_{2, \lambda}}{\gamma_{1, \lambda}+\rho M_{1, \lambda}} c_{2}
$$

if $\rho \notin]-1,0[$, or

$$
c_{2}=-\frac{\gamma_{1, \lambda}+\rho M_{1, \lambda}}{M_{2, \lambda}+\rho \gamma_{2, \lambda}} c_{1}
$$

if $\rho \geq-1$. This show that if $\lambda-A$ is injective, we necessarily have that just one of the numbers $M_{1}$ and $M_{2}$ must be finite and consequently we obtain the validity of condition (2.8) or (2.9).

Remark 2.2. In general, we cannot expect the validity of the dissipativity property even in the cases where $\lambda-A$ is injective for every $\lambda>0$.

To show this, consider the operator

$$
\left.A u(x)=x(1-x) u^{\prime \prime}(x)+u^{\prime}(x), \quad x \in\right] 0,1[
$$

on the domain $D_{-1}(A)$. In this case, choosing $x_{0}=1 / 2$, we have $W(x)=x /(1-x)$ and $R(x)=(2 x-1) /(1-x)$.

Hence $A$ satisfies (2.8); moreover, for $u \in C^{2}([0,1])$ we have $u \in D_{-1}(A)$ if and only if $u^{\prime}(0)=$ $u^{\prime}(1)$.

Thus, if $c>2$ and $\lambda>0$, the function $u(x)=c-x$ is in $D_{-1}(A)$ and satisfies $\lambda\|u\|=\lambda c$, while $A u(x)=1$ and consequently $\|\lambda u-A u\|=\lambda c-1$; hence, the operator $\left(A, D_{-1}(A)\right)$ is not dissipative.

A similar discussion can be carried out to show that $\left(A, D_{\rho}(A)\right)$ does not satisfy in general the positive minimum principle. If we consider the above example, the function $u(x)=x$ is positive on $[0,1]$ and vanishes at 0 , while $A u(x)=-1<0$.

According to the work of Feller [7], if $A$ satisfies condition (2.7) and $\rho \neq 0$, we may not expect that $\left(A, D_{\rho}(A)\right)$ generates a (positive) contraction semigroup.

However we can also obtain the surjectivity of the operator $\lambda-A$ for every $\lambda>\lambda_{0}$.

Proposition 2.2. Assume that $\rho \neq 0$ and that $\left(A, D_{\rho}(A)\right)$ satisfies condition (2.7) and one the conditions (2.8) or (2.9). Then there exists $\lambda_{0} \geq 0$ such that $\lambda-A$ is surjective for every $\lambda>\lambda_{0}$. Moreover, for every $\lambda>\lambda_{0}$ and every $f$ and $f \in C(\bar{I})$, the unique solution $u \in D_{\rho}(A)$ of $(\lambda-A) u=f$ satisfies the estimate

$$
\|u\| \leq \frac{1+2|\rho|}{\lambda-\lambda_{0}}\|f\|
$$

Finally, if condition (2.8) holds, we can take $\lambda_{0}=0$ if and only if $\rho \in[-1,+\infty[$ while, if condition (2.9) holds, we can take $\lambda_{0}=0$ if and only if $\left.\left.\rho \in\right]-\infty,-1\right] \cup[0,+\infty[$.

Proof. Let $\lambda_{0}$ as in Theorem 2.1 and fix $f \in C(\bar{I})$. If (2.8) holds, the function $\chi_{2, \lambda}$ is bounded and therefore the equation $\lambda u-A u=f$ admits infinite bounded solutions

$$
u=u_{f}+c \chi_{2, \lambda}
$$

depending on the constant $c$, where $u_{f}$ is defined by considering in (2.5) the Green's function $G_{\chi_{1, \lambda}, \chi_{2, \lambda}}$ relative to $\chi_{1, \lambda}$ and $\chi_{2, \lambda}$. 
Moreover, since $\lim _{x \rightarrow r_{1}} A u_{f}(x)=0$, we have

$$
\lim _{x \rightarrow r_{1}} A u(x)+\rho \lim _{x \rightarrow r_{2}} A u(x)=c \lambda M_{2, \lambda}+\rho A u_{f}\left(r_{2}\right)+c \rho \lambda \gamma_{2, \lambda}
$$

and therefore we obtain a unique solution in $D_{\rho}(A)$ corresponding to

$$
c:=-\frac{\rho A u_{f}\left(r_{2}\right)}{\lambda\left(M_{2, \lambda}+\rho \gamma_{2, \lambda}\right)} .
$$

Hence, the function

$$
u:=u_{f}-\frac{\rho A u_{f}\left(r_{2}\right)}{\lambda\left(M_{2, \lambda}+\rho \gamma_{2, \lambda}\right)} \chi_{2, \lambda}
$$

is the unique solution of $(\lambda-A) u=f$ and this completes the proof of the surjectivity of $\lambda-A$. As regards to estimate (2.17), let $f \in C(\bar{I})$ and consider the unique solution $u:=(\lambda-A) f$ the unique solution of $(\lambda-A) u=f$ in $D_{\rho}(A)$ given in (2.20). We already know that the operator $A$ generates a $C_{0}$-semigroup of positive contractions on the following domain (see [9, Theorem 4])

$$
D_{V M}:=\left\{u \in C(\bar{I}) \cap C^{2}(I) \mid A u \in C(\bar{I}), \lim _{x \rightarrow r_{1}} A u(x)=0\right\} .
$$

Hence the equation $(\lambda-A) v=f$ has a unique solution $v:=R(\lambda, A) f \in D_{V M}(A)$ and further $\|v\| \leq\|f\| / \lambda$. From the equality $A R(\lambda, A)=\lambda R(\lambda, A)-I$ and since $M_{2, \lambda}+\rho \gamma_{2, \lambda}>0$ for every $\lambda>\lambda_{0}$, we get

$$
\begin{aligned}
\|u\| & \leq\|v\|+\frac{|\rho|\|A R(\lambda, A) f\|}{\lambda\left(M_{2, \lambda}+\rho \gamma_{2, \lambda}\right)} M_{2, \lambda} \leq \frac{\|f\|}{\lambda}+\frac{|\rho|\|R(\lambda, A) f\|}{1+\rho \gamma_{2, \lambda} / M_{2, \lambda}}+\frac{|\rho|\|f\|}{\lambda\left(1+\rho \gamma_{2, \lambda} / M_{2, \lambda}\right)} \\
& \leq \frac{\|f\|}{\lambda}+\frac{|\rho|\|f\|}{\lambda}+\frac{|\rho|\|f\|}{\lambda}=\frac{1+2|\rho|}{\lambda}\|f\| \leq \frac{1+2|\rho|}{\lambda-\lambda_{0}}\|f\|
\end{aligned}
$$

which completes the proof in the case where condition (2.8) holds. An analogous reasoning can be carried out if condition (2.9) holds. The last part is a consequence of Theorem 2.1.

Assume that $\rho \neq 0$ and that $\left(A, D_{\rho}(A)\right)$ satisfies condition (2.7) and one of the conditions (2.8) or (2.9). From the preceding results it follows that $\left(A, D_{\rho}(A)\right)$ is closed (Lemma 2.1) and by Theorem 2.1 and Proposition 2.2 we find $\lambda_{0} \geq 0$ such that for every $\lambda>\lambda_{0}$ the operator $\lambda-A$ is invertible and its inverse satisfies the estimate

$$
\left\|(\lambda-A)^{-1}\right\| \leq \frac{1+2|\rho|}{\lambda-\lambda_{0}} .
$$

The last estimate does not ensure that that the operator $\left(A, D_{\rho}(A)\right)$ generates a $C_{0}$-semigroup in $C(\bar{I})$. Moreover, the better case can be obtained by setting $\rho=0$ and in this case we have the generation of $C_{0}$-semigroup from the classical Hille-Yosida theorem.

Many authors have studied the possibility of approximating the solutions of evolution problems by using the iterates of suitable approximation processes (see [1,2] for a complete description of these methods). Using the results in this paper we can approximate the solution of similar problems when different boundary conditions are assigned.

\section{ACKNOWLEDGMENTS}

Work performed under the auspices of G.N.A.M.P.A. (INdAM). 


\section{REFERENCES}

[1] F. Altomare, M. Campiti, Korovkin-type Approximation Theory and its Applications, De Gruyter Studies in Mathematics 17, W. De Gruyter, Berlin-New York, 1994.

[2] F. Altomare, M. Cappelletti Montano, V. Leonessa and I. Raşa, Markov Operators, Positive Semigroups and Approximation Processes, de Gruyter Studies in Mathematics 61, de Gruyter, Berlin/Boston, 2014.

[3] A. Attalienti, M. Campiti, Semigroups generated by ordinary differential operators in $L^{1}(I)$, Positivity 8 (1) (2004), $11-30$.

[4] M. Campiti, S. P. Ruggeri, Approximation of semigroups and cosine functions in spaces of periodic functions, Applicable Analysis 86 (2) (2007), 167-186.

[5] Ph. Clément, C. A. Timmermans, On $C_{0}$-semigroups generated by differential operators satisfying Ventcel's boundary conditions, Indag. Math. 89 (1986), 379-387.

[6] K.-J. Engel, R. Nagel, One-parameter semigroups for linear evolution equations, Graduate Text in Mathematics 194, Springer, New York, 2000.

[7] W. Feller, The parabolic differential equations and the associated semi-groups of transformations, Annals of Math. 55 (3) (1952), 468-519.

[8] P. Mandl, Analytical treatment of one-dimensional Markov processes, Die Grundlehren der mathematischen Wissenschaften in Einzeldarstellungen 151, Springer-Verlag, Berlin-Heidelberg-New York, 1969.

[9] C. A. Timmermans, On $C_{0}$-semigroups in a space of bounded continuous functions in the case of entrance or natural boundary points. In: Gòmez-Fernandez J.A., Guerra-Vázquez F., Lòpez-Lagomasino G., Jiménez-Pozo M.A. (eds). Approximation and Optimization, Lecture Notes in Mathematics, 1354. Springer, Berlin, Heidelberg, 2006.

\section{UNIVERSITY OF SALENTO}

Department of Mathematics and Physics "E. De Giorgi"

VIA PER ARNESANO, LECCE, ITALY

E-mail address: michele.campiti@unisalento.it 